Proceedings

\title{
The Birth and Evolution of Symbolic Information ${ }^{\dagger}$
}

\author{
José Monserrat Neto \\ Department of Computer Sciences, Universidade Federal de Lavras, Lavras 37200-900, Brazil; \\ jose.monserrat.neto@gmail.com; Tel.: +55-35-988313319 \\ † Theoretical Information Studies (TIS) Conference, Berkeley, California, USA, 2-6 June 2019. \\ Published: 13 May 2020
}

\begin{abstract}
This work presents the main points of Deacon's theory about the emergence of human language, which are summarized as follows: (1) a relaxed selection processes with the evolution of cooperative social life; (2) the development of first simple symbolic information systems of earlier hominids; (3) their long evolution to language over 2 million years, in a bio-cultural co-evolution of both language and the brain. It examines the main foundations of Deacon's theory, how these have been deepened gradually, and the hypotheses concerning the first symbolic systems and possible ways that they evolved to language and human species.
\end{abstract}

Keywords: language; evolution; symbolic system; emergence

\section{Introduction}

In The Symbolic Species [1], Deacon presented the core lines of an evolutionary theory of the symbolic communication of language, unique to humans. This paper presents the general line of his theory, the main points of its foundation, and the birth of the first symbolic systems. Deacon's theory is 23 year old. Surprisingly, it is not well known outside of restricted academic circles. So, this paper also aims to disseminate his theory.

\section{Transition from Hominids to Humans}

The basic idea of the transition in Symbolic Species [1] (chapter 12) is that symbolic capacity was developed in earlier hominids around 2 million years ago (mya) in an increasingly cooperative social environment. Deacon argues that symbolic capacity could not emerge later, as is supposed by many current theories of human evolution, because language is too complex to be developed in, for instance, hundreds of thousands of years. There would not be enough time for brain structures and symbolic systems to evolve into the complex language that humans use today. So, the basic idea is that, from a more cooperative social life, the first very simple symbolic systems emerged around 2 mya, and, then, through a long co-evolution between these first symbolic systems and the many structures of the brain and body, they have gradually evolved into our current languages.

\section{Foundations of Deacon's Theory}

In Symbolic Species [1], Deacon starts with observing an oddity: "There is no simple language today". Language is a very refined symbolic system with no counterpart in animals. To explain such a rarity, Deacon draws upon a version of Peircean semiotics theory [2], the idea that biological development is emergent, and on a revised version of natural selection theory.

Confusion over the nature of symbolic references is at the core of the challenge to understand language. In Peircean semiotics, communication is structured in nested hierarchical levels: iconic, indexical and symbolic [2]. Thus, there are two kinds of communication: indexical communication, structured over iconic references and used by elephants, dolphins, and monkeys, for instance; and 
symbolic communication, structured over indexical references. Animals do not utilize symbolic communication because it is difficult to learn and use; that is, it is very counter-intuitive and has demanding requirements of brain memory and processing. There are issues with the current view of symbol conventionality, namely that it is not a critical determiner of symbolic reference. Signs of an iconic smile and indexical toilet are conventionalized icons and indexes too. Symbolic reference requires conventionalized sign vehicles in order to dissociate sign vehicle properties from referent properties. Therefore, symbols are doubly conventional. Such confusions have blocked progress in grasping language and have led to unsolvable problems: the existence of a Universal Grammar, its mysterious innateness and evolvability; and the symbol grounding problem, or how arbitrary symbols are grounded the first time [3].

An experiment with chimpanzees clarifies Deacon's argument. Most of the experiments show chimpanzees' ability via lexigrams to interpret indexically, not symbolically. But in one experiment scientists have proven chimpanzees' ability to make the transition from indexical to symbolic interpretation. That was set up with only six lexigrams: two foods, two drinks and two verbs ("give" and "pour"). In the transition, as the chimps engaged in thousands of trials, what was discovered was a symbolic system of classification (liquid and solid). Once the chimps learned this, adding a new vocabulary of another lexigram (e.g., a new food) became much easier. Two things must also be noted: the symbolic system is not imposed, but emergences via chimps' experiences; and, if they are able to learn it, the earlier hominids should also have been able to do so.

The engineering fallacy is what is behind the faulty view of symbols; that is, the habit of thinking of organism structures and brain functions in engineering terms. This view sees natural selection as a designer, and organisms as its designed products. But an organism is not assembled, it is selfdetermined. Its components develop from an initially undifferentiated whole into fully differentiated parts. This is analogous to the linguistic fallacy. Language is a spontaneously evolved emergent adaptation, not a formal computational system. Its structure does not derive from either innate or social instruction, but rather from self-organization and selection. The neurological processing of sentence structure is more analogous to embryonic differentiation than to an algorithmic computation [3].

From the engineering perspective, the design of organism is passed through generations in genetic codes that determine the making of the design. In this view, changes are seen only by chance mutation of DNA. This role is, however, only half of the story. From the emergent perspective, DNA sets up biological points that channel the self-development of organisms. In Symbolic Species, Deacon draws on two mechanisms: Baldwin's and Waddington's. The former shows how the context in which natural selection happens can be modified by organism behavior, and such change can generate new selection pressures. The later shows in turn how the new behaviors and their changed selection pressures can be genetically assimilated through mutations. The beaver species provides an example: in building dams, new behavior changes the ecological context in that beaver evolves through new selection pressures, and this can lead to genetic changes to beavers' teeth and tails, for instance.

\section{Further Developments of Deacon's Theory Foundations}

Since 1997, Deacon and colleagues have deepened the foundations of his theory. Among these are: (a) the reformulation of some aspects of natural selection theory; (b) relaxed selection in evolution; (c) the origin of teleodynamics of life; (d) the process underlying hierarchic transitions in evolution; (e) bio-semiotic reformulation of the Shannonian theory of information [4]; and, (f) studies on the metaphysics of emergent dynamics [5].

Though important, Baldwin's and Waddington's mechanisms are not sufficient to explain the emergence of new traits, like the association of cells in bodies and the altruistic behaviors of animals. The theories of kin selection and group selection are unable to explain how associations arise in the first place, only how they are maintained. Deacon proposes a third mechanism: the loss of autonomy through relaxed natural selection in individual units. In the process of losing their autonomy, cells 
become genetically "addicted" to a multicellular organism, and animals become socially "addicted" to a co-dependent group of a species [5].

In Symbolic Species, there was no theory of self-emergence implicit in the theory of language evolution. In Incomplete Nature [6], Deacon shows in a rigorous way how life and mind can emerge from simple physical-chemical processes and give rise to the teleodynamics of life. His ideas are, however, tricky to grasp easily. Fortunately, there is an intuitive introduction to his approach in Neither Ghost nor Machine [7].

Deacon proposes a process underlying the hierarchic transition in evolution, summarized in four steps: (1) duplication, (2) relaxed selection, (3) degradation, and (4) new synergies. The process is initiated by the appearance of functional redundancy: duplication [8]. In the second and third step, relaxed selection conditions predispose the degradation of duplicate sources, so long as at least one maintains its functional integrity. Subsequently, the redundant variant forms may interact to produce complementary effects, replacing the original constraint with a distributed alternative, the new synergy. As this gives rise to advantageous synergistic functions and degrades previous precursors, this will introduce novel selection pressures to stabilize the new higher-order adaptation $[9,10]$.

\section{Paradigmatic Case of the Bird Bengales Finch}

The evolution of the Bengales finch from its wild cousin the White Rump Munia helps us to glimpse the transition to language [5,8]. A study compares a wild finch to a domesticated breed of the same species that has been bred for coloration for 250 years. Surprisingly, in the absence of breeding for singing and shielded from natural and sexual selection, the finches produced increased song complexity and greater involvement of social learning in song development. How could an increase in the complexity of song structure and neural systems for producing song have evolved without overt selection to get these traits? The prolonged domestication masked a selection that is maintained in the wild highly canalized control over song production. Reducing environmental pressures and sexual selection effects led to degradation of previously genetic constraints and allowed increased variability. If in 250 years the finch could undergo such a change, what could happen in 2 million years in the evolution of humans? However, the genetic degradation enabled by the relaxed selection of a new functional adaptation-symbolic communication, for instance-is not a cause of a new adaptation. Degradation via relaxed selection is just a source of highly biased combinatorial variations that can, once the new adaptation has emerged, enhance the new useful adaptation under the influence of selection. Therefore, how did the symbolic adaptation have emerged in the first place?

\section{Special Conditions in the Birth of the First Symbolic System}

The social niche of the first hominids was special. There is evidence of relaxed selection around 2 mya. The use of stone tools by upright hominids probably induced the processes of relaxing the natural selection pressures. It led to a reduction in masticatory adaptations and a reduction of sexual dimorphism with less intense male-male sexual competition. Yet, the new social niche of the first hunting hominids was unstable. Among all cases of hunting species, the hominid was the only one where there was no alpha hunter who mated with all females, as happens with lions and wolfs, etc. Deacon hypothesizes the first symbolic system could have been the "mate contract", in that through collective rituals the hominids started to communicate and regulate symbolically who could mate with whom, reinforcing a more monogamous life, at least for some time. However, an important caveat is that there is no need for the first symbolic system to have been the "mate contract". In his theory, the only need is that the first symbolic systems should have been as simple as possible and attempted to solve a hominid problem that could not be sorted out except by a symbolic means. In the case of the six lexigrams, what emerges in the long training is a simple symbolic system that distinguishes liquid from solid. In turn, in the case of the "mate contract", what emerges from the repeated rituals of the whole hominid group is a symbolic system that distinguishes between who is married and who is available to mate. 


\section{Key Changes from First Symbolic Systems to Language}

Whatever the first symbolic systems were, they could be under the influence of selection and could enhance the new symbolic adaptation, and in 2 million years they could have transformed into our language. In the case of the finches, relaxed selection produced a progressive degradation of circuits that contribute tight constraints on the motor patterning that specifies song structure. With relaxing sexual selection pressures, influences that were previously inhibited could play a role in biasing song formation and learning. In the human case, the symbolic adaptations of language required vocal flexibility and the decoupling of vocalizations from specific cognitive-emotional states, and several other adaptations from a greater relaxed selection environment (fire and cooking practices), like the specialization of brain areas, expansion of memory and of the prefrontal cortex to better control symbol use, the fine control of breath, the descent of vocal tract, and new forms of integration between symbols and emotions [11,12]. To sum up, language and the brain co-evolved in a new symbolic niche and developed to fit the symbolic capabilities of the child, who learns language in a quick and easy way. As Deacon says in Symbolic Species, the first symbolic systems have turned into the "tail that wags the dog".

\section{Conclusions}

I have summarized the main points of Deacon's theory on the origin of language. His theory is not yet complete. Several steps in the evolutionary process have still to be established properly. Even so, I think his theory has a great advantage. It opens a rigorous pathway to help face the current troubles of language evolution. It avoids the long-standing unsolvable problems of current theories, such as the innate grammar or the innate language modules, appearing suddenly by miraculous genetic mutations.

\section{References}

1. Deacon, T. The Symbolic Species - The Co-evolution of Language and The Brain; W. W. Norton \& Company: New York, NY, USA, 1997.

2. Deacon, T. The Symbol Concept. In The Oxford Handbook of Language Evolution; Tallerman, M., Gibson, K., Eds.; Oxford University Press: Oxford, UK, 2011; pp. 393-405.

3. Deacon, T. The Emergent Process of Thinking as Reflected in Language Processing. In Thinking Thinking: Practicing Radical Reflection; Schoeller, D., Saller, V., Eds.; Verlag Karl Alber: Freiburg im Breisgau, Germany, 2016; pp. 135-158.

4. Deacon, T. Information and Reference. In Representation and Reality: Humans, Animals and Machines; DodigCrnkovic, G., Giovagnoli, R., Eds.; Springer: Berlin, Germany, 2017; pp. 3-15.

5. Deacon, T.; Cashman, T. Steps to a Metaphysics of Incompleteness. Theol. Sci. 2016, 14, 401-429.

6. Deacon, T.W; Hui, J. The Evolution of Altruism via Social Addiction. Proc. Br. Acad. 2009, 158, 181-203.

7. Deacon, T. Incomplete Nature - How Mind Emerged from Matter; W. W. Norton \& Company: New York, NY, USA, 2012.

8. Sherman, J. Neither Ghost nor Machine-The Emergence and Nature of Selves; Columbia University Press: Berkeley, CA, USA, 2017.

9. Deacon, T. Process Underlying the Hierarchic Transition in Evolution. Unpublished, 2018.

10. Deacon, T. A Role for Relaxed Selection in the Evolution of the Language Capacity. Proc. Natl. Acad. Sci USA 2010, 107, 9000-9006.

11. Deacon, T. Aesthetic Faculty. In The Artful Mind: Cognitive Science and the Riddle of Human Creativity; Turner, M., Ed.; Oxford University Press: New York, NY, USA, 2006; pp. 21-53.

12. Deacon, T.; Cashman, T. The Role of Symbolic Capacity in the Origins of Religion. J. Study Relig. Nat. Cult. 2009, 3, 490 . 